\title{
In the hands of users with Learning Disabilities: Co-Designing Tangible Users Interfaces for Mental Wellbeing
}

This paper was downloaded from TechRxiv (https://www.techrxiv.org).

\section{LICENSE}

CC BY-SA 4.0

SUBMISSION DATE / POSTED DATE

$12-01-2021 / 13-01-2021$

\section{CITATION}

Kanjo, Eiman; Woodward, Kieran; Harold, Gordon; McGinnity, Martin; Brown, David (2021): In the hands of users with Learning Disabilities: Co-Designing Tangible Users Interfaces for Mental Wellbeing. TechRxiv. Preprint. https://doi.org/10.36227/techrxiv.13562693.v1

$\mathrm{DOI}$ 


\title{
In the hands of users with Learning Disabilities: Co-Designing Tangible Users Interfaces for Mental Wellbeing
}

\author{
KIERAN WOODWARD, Nottingham Trent University, UK \\ EIMAN KANJO, Nottingham Trent University, UK \\ DAVID J BROWN, Nottingham Trent University, UK \\ T.M. MCGINNITY, Nottingham Trent University, UK \\ GORDON HAROLD, University of Cambridge, UK
}

Involving and engaging people with learning disabilities on issues relating to their mental wellbeing can be challenging. This research explores how participatory design techniques and principles can be used to engage people with learning disabilities in designing technological solutions relevant to them that could monitor and aid their mental wellbeing. Specifically, we explore methods used in a series of co-design workshops to engage people with learning disabilities in the use of tangible interfaces for mental wellbeing. A variety of designs, sensors and interventions were explored during the workshops resulting in the development of multiple mental wellbeing interfaces. Furthermore, data collection trials using the developed interfaces have demonstrated the potential to collect real-world labelled sensor data. The co-design approach adopted for the design of the mental wellbeing tangible interfaces ensured that effective and suitable devices have been developed.

\section{INTRODUCTION}

Individuals with learning disabilities often experience mental wellbeing challenges but these are often overlooked and attributed erroneously to their learning disability (diagnostic overshadowing) or classed as challenging behaviour [24]. Diagnostic overshadowing often results in a significant impact on an individual's likelihood of engaging with mental healthcare systems. Furthermore, many individuals with learning disabilities find it challenging to express the emotions they experience. Advances in sensors, embedded systems, edge computing and machine learning [75] can address these challenges by helping those with a learning disability to understand and express their emotions to others.

Recent advances in sensors, batteries and processing have resulted in an increase of pervasive computing technologies. However, many interfaces only include a limited range of sensors such as those measuring motion and Heart Rate (HR) and infrequently record this data, resulting in data that may not capture all of the wellbeing states experienced by users and is not sufficient to train machine learning classifiers. Furthermore, the vast majority of current interfaces that monitor wellbeing do not include methods that can serve as intervention to promote the regulation of negative emotions [25]. The possibility of a device to accurately monitor real-world wellbeing using multi-modal sensor data presents the possibility for automated interventions that are applied once poor wellbeing is inferred.

Tangible User Interfaces (TUIs) describe physical objects that are able to translate user actions into input events in the computer interface. TUIs enable people to interact with digital data through physical objects and are ideal to embed all of the necessary sensors and microprocessor to translate user actions in the real-world [65]. TUIs present an opportunity to develop novel devices going

Authors' addresses: Kieran Woodward, kieran.woodward@ntu.ac.uk, Nottingham Trent University, Nottingham, UK; Eiman Kanjo, eiman.kanjo@ntu.ac.uk, Nottingham Trent University, Nottingham, UK; David J Brown, Nottingham Trent University, Nottingham, UK; T.M. McGinnity, Nottingham Trent University, Nottingham, UK; Gordon Harold, University of Cambridge, Cambridge, UK. 
beyond traditional wearables to enable unique interaction methods and encourage continued engagement.

We envisage a successful TUI that will enable mental wellbeing to be automatically monitored using multi-modal sensors embedded within the device. Once wellbeing has been inferred, it further enables the possibility of applying digital feedback to act as interventions aiming to automatically and gradually regulate emotions and improve wellbeing in real-time [77]. However, before it is possible to classify wellbeing, machine learning models must first be trained using large, labelled datasets. Even though real-world physiological sensor data is notoriously challenging to collect due to body movements impacting sensor data [16] and the challenging propositions of labelling at the point of collection, it is vital to collect data in different settings, and not just controlled 'synthetic' data from experiments to ensure the model and device can successfully operate in the "wild" (naturalistic settings).

To ensure TUIs are effective and usable by individuals with learning disabilities, co-design workshops have been conducted to design the interfaces and explore different sensing and feedback mechanisms with participants from the Nottingham Interactive Community for Education Research (NICER) group at Oak Field school, Nottingham, UK. The group is formed of adults with a range of learning disabilities who have a wide range of experience in evaluating enabling technologies including virtual environments and serious games.

The feedback from the workshops has been used to design and develop a range of tangible interfaces designed for both children and adults, embedding multi-modal sensors to monitor mental wellbeing. Tangible labelling techniques were also co-designed and were used by participants to overcome the traditional challenges when collecting real-world labelled mental wellbeing data A series of focus groups followed to gather additional feedback further guiding the labelling approaches and commencing real-world data collection trials.

The remainder of this paper is organised as follows: Section 2 provides a literature review on co-design, TUIs and affective computing; Section 3 describes the co-design methodology to design and develop the interfaces and analysis of the workshops; Section 4 explores the design revisions of the interfaces; Section 5 describes the data collection method and findings; Section 6 evaluates the TUIs using focus groups and describes a revised data collection; Section 7 provides the discussion and Section 8 concludes and offers recommendations.

\section{BACKGROUND}

\subsection{Co-Design}

Co-design is closely connected with participatory design and is the methodology for actively engaging people directly involved in an issue, place or process in its design allowing them to make a meaningful contribution to the design process [15], [9], [30]. Co-design enables the reduction of the gap in knowledge between end users and researchers, allows non-designers to become equal members of the design team ensuring designer subjectivity is removed and the technologies developed are suitable for the target population[68], [52]. During the process, design tools are used to empower all of the participants to facilitate a 'joint inquiry' where 'problem and solution coevolve' [63]. Co-design brings many benefits to the design of the project by helping the researcher better understand the challenges faced by users and any potential solutions [64], [60].

Co-designing helps solve real-world problems by bringing together people from different backgrounds into the design process, resulting in more inclusive design solutions. However, to work most effectively it is important to select appropriate methods and ways of working which need to match the project being designed and the potential users' capabilities and limitations. Co-design 
methods help make things that are normally unobservable through traditional interviews and focus groups available as resources for design [69], [20], [70], this can be achieved by:

- using visual, creative methods [49].

- physically making things helping people to explore, verbalise, remember and imagine [52].

- creating and telling stories helping to put things into context and providing a central way of sharing and communicating. Story sharing can be visual, verbal or include role play [49].

Co-design can be used to promote the inclusion of people living with disabilities when designing new solutions by including their personal experiences, making them more likely to take ownership of the final outcome [53]. People with learning disabilities may face barriers such as communication challenges when being involved in the co-design of new assistive technologies, resulting in codesign techniques needing to be modified to fit with participants' abilities. To help reduce the challenges faced, a set of guidelines have previously been devised for co-designing with people who have autism spectrum disorders [21].

Co-design has previously been used to engage individuals with cognitive disabilities in successfully designing a picture-based remote communication system helping the participants move from passive onlookers to active participants[19]. Similarly, co-design workshops have engaged people with assisted living needs to develop technologies and services for new care solutions [71] and engaging autistic children in co-designing technologies [61]. Furthermore, co-design has been used to develop mobile applications with adults who have intellectual disabilities, highlighting that prototypes were required to deepen user engagement [59]. Overall, while involving users with learning disabilities in the design process produces additional challenges such as additional ethical considerations [62], it is imperative to ensure the solutions developed meet the needs of the potential users.

\subsection{Tangible User Interfaces}

TUIs present many opportunities to sense wellbeing by enabling individuals to interact naturally with digital information by manipulating physical objects [76], [75]. TUIs can take any form enabling them to ubiquitously become part of everyday interactions in comparison with graphical user interfaces. This type of embodiment enables people to have stronger emotional attachments with physical devices and interactive experiences than merely digital interfaces [40], [43].

TUIs for mental wellbeing can be used to enable the active self-reporting of current state of wellbeing using novel interaction techniques or a passive approach can be used to collect objective sensor data to infer wellbeing [78]. Many previous wellbeing TUIs have adopted an active approach such as recording emotions by squeezing an electronic ball [22]. Similarly, Subtle Stone [7], allowed users to record their emotions as colours. Participants liked the simplicity of representing emotions as colours, and the ability to privately share their emotions with trusted people but not the general population providing anonymity.

Alternatively, Grasp, which was been tested in a dentist's office [27] measured how much force was exerted when participants squeezed and transmitted this data to a mobile app. By utilising force sensors to measure touch, Grasp enabled users to quickly and easily record their stress levels in realtime without self-reporting. Sensors such as force sensors and accelerometers to detect touch and motion have been shown to provide an intuitive method of interaction for tangible interfaces [27], [37], [44]. These sensors show the increased opportunities tangible interfaces present over devices such as smartphones by embedding a range of sensors to infer mental wellbeing. It is also possible to collect data using a hybrid approach where the self-reporting of emotions is combined with the passive collection of sensor data. Mood TUI [54], combined self-reporting with the recording of data from users' smartphones including location, phone use and HR. The results demonstrate 
the requirement of an accessible design when developing tangible interfaces as while participants found Mood TUI exciting they thought it was physically too large to continue using for extended periods of time.

\subsection{Affective Computing}

Recent development in machine learning are helping increase the accuracy in which mental wellbeing can be classified from sensory data [10]. There are numerous sensors that can be used to classify mental wellbeing when combined with sufficiently trained machine learning classifiers. A combination of small, reliable sensors and machine learning could result in interfaces that can accurately infer real-world mental wellbeing in real-time which may be extremely beneficial for those with learning disabilities who often find it challenging to communicate their emotions.

Non-invasive physiological sensors present a significant opportunity to monitor mental wellbeing. However, many physiological sensors traditionally used to collect mental wellbeing data such as electroencephalograms [4] are extremely challenging to use outside of controlled laboratory experiments. Electrodermal Activity (EDA) and Heart Rate Variability (HRV) sensors are especially beneficial when classifying real-world wellbeing as they are small sensors that directly correlate to the sympathetic nervous system [56] [55] [1]. Both EDA and HRV have been used in a device that aimed to measure stress [29]. The device took measurements over a five minute period and was successfully able to detect stress levels with an accuracy of $97.4 \%$. Similarly, a personalised transfer learning approach has inferred mental wellbeing with $93.9 \%$ accuracy across 3 participants using HRV and EDA sensor data [73]. The results showed HRV and EDA are highly correlated when inferring stress, suggesting they are appropriate sensors to use in mental wellbeing tangible interfaces.

EDA, skin temperature, motion and phone usage data has previously been used to infer stress resulting in $81.4 \%$ accuracy, outperforming other machine learning models such as linear regression and support vector machine [67]. A 1-dimensional CNN has also been trained using EDA and blood volume pulse data to classify relaxation, anxiety and excitement[39], achieving between $70 \%$ and $75 \%$ accuracy.

Recently, Convolutional Neural Networks (CNN) Long Short-Term Memory (LSTM) models have been combined to infer emotions [33]. Physiological and environmental data including EDA, Heart Rate (HR), body temperature and noise was used to train the model which outperformed traditional deep learning models by around 20\%. Deep learning demonstrates the ability to infer mental wellbeing, although it initially requires a large labelled dataset to train the models which can be challenging to collect [58].

Once mental wellbeing has been classified it is possible to automatically apply interventions as on-device feedback. There are numerous feedback actuations including visual, auditory, vibrotactile and temperature that can be considered for affective interfaces [2]. Good vibes [34], the Relax! pen [17] and Doppel [6] all used haptic feedback to reduce stress. These studies show participants remained less stressed when using the devices compared with control groups when in stressful situations demonstrating the benefits of haptic feedback in improving mental wellbeing. Haptic feedback can further be utilised as a breathing pacer. Miri [41] found that the development of a personalised routine where users find the breathing frequency comfortable was more important in relaxing users than the placement of the feedback itself. This demonstrates the benefits of haptic feedback to passively calm users and actively aid relaxing breathing practices.

Many existing approaches have not classified wellbeing to issue feedback but instead mirrored physiological reactions back to users for their reflection [47]. Visual feedback in the form of flexible wrist-worn displays have been developed enabling users to view real-time representations of their 
physiology [66]. However, the use of a thermochromic display resulted in the visualisations being ambiguous and potential privacy concerns as other people could easily view the wearer's emotions.

Somaesthetics has also been used as a method of combining bodily experiences with aesthetic appreciation presenting many opportunities for expressing mental wellbeing [72]. The Breathing Light [31] created an enclosed space where light dynamically changed with the user's breathing patterns enabling deep reflection. The Breathing Light provided a more calming alternative for visual feedback but may be challenging to adopt in real-world environments. Similarly, BrightHearts is a biofeedback mobile app that changed patterns, colour and sound with the user's HR, helping people become self-aware of their wellbeing and assisting them in relaxing [35]. Somatics has additionally been used to translate EDA data into felt experiences through changes in temperature [3]. Wearer's of the device found the cooling sensation triggered by changing arousal levels to be pleasant. The use of varying temperatures as feedback is a novel method that when paired with additional sensors to better monitor wellbeing could provide subtle calming feedback that may help improve wellbeing.

Alternatively, therapy animals have been used to provide biofeedback. Therapy animals such as Paro [57] are commonly used by the elderly to reduce loneliness. Force sensors enable the location and force of users' touch to be used to make the feedback relevant to the user input. Paro has provided effective therapy by helping to reduce stress in a day service centre for elderly adults [5], increasing users' social interactions and improving users' reactions to stress [57].

This research demonstrates the potential impact tangible interfaces can have when inferring emotions and providing real-time feedback. However, much of the existing research has either only explored the classification of wellbeing or the application of interventions. There has been little research on the co-design and development of tangible interfaces for mental wellbeing data collection, inference and real-time feedback.

\section{CO-DESIGN WORKSHOPS}

Two co-design workshops have been conducted with the aim of designing mental wellbeing tangible interfaces. Each co-design workshop was conducted with a primary researcher and an experienced facilitator with many years experience in running co-design workshops. All participants were members of the NICER group with varying physical and cognitive disabilities. Co-designing with people with learning disabilities presents additional challenges - such as issues regarding informed consent and the nature of their cognitive disability, including communicational and memory issues, which might challenge their full participation. However, it is imperative for the voice of end users to be heard and many of the challenges can be overcome by developing a co-design methodology catering for the requirements of people with learning disabilities.

The aim of the workshops was not to achieve one design with specific sensors, instead we expected a range of different devices to be proposed to suit the needs of all potential users. The co-design workshops aimed to explore the following research questions:

(1) What designs would users prefer for mental wellbeing tangible interfaces?

(2) What are the different sensors that users would want to interact with?

(3) How do users believe on-device feedback could help improve their mental wellbeing?

(4) How can the interfaces be used to collect real-world data?

The following participants were involved within the two co-design workshops. The first workshop comprised of six participants; four males and two females who have previously been involved with multiple research projects and are experienced co-designers. The second workshop comprised of 8 participants; five males and three females who again have are experienced co-designers. Table 1 
shows the characteristics of the participants including those who have Williams Syndrome (WS), Down Syndrome (DS) and Autism along with gender and level of learning disability.

Table 1. Participant characteristics

\begin{tabular}{|l|c|c|r|r|r|}
\hline & & Total $(N=9)$ & WS $(N=2)$ & DS $(N=4)$ & Autism $(N=1)$ \\
\hline Mean age at 2020 in years & & 34.4 & 38.5 & 39.75 & 18 \\
$(\mathrm{SD})$ & & $(9.1)$ & $(0.71)$ & $(7.3)$ & \\
\hline Gender $N(\%)$ & Male & $6(66.7)$ & $2(100)$ & $2(50)$ & $1(100)$ \\
& Female & $3(33.3)$ & $0(0)$ & $2(50)$ & $0(0)$ \\
\hline Level of learning disability $N(\%)$ & Moderate & $1(11.1)$ & $0(0)$ & $1(25)$ & $0(0)$ \\
& Severe & $8(88.9)$ & $2(100)$ & $3(75)$ & $1(100)$ \\
\hline
\end{tabular}

\subsection{Methodology}

A common issue with the development of mental wellbeing technological solutions is the lack of ethical considerations [48]. To ensure the co-design workshops were inclusive for participants with learning disabilities and caused no harm to participants all discussions were short and a range of interactive tasks were designed to increase engagement. The following five affordances used within the co-design workshops were designed to support participants' decisions and compensate for their disabilities based on established methods and the extensive previous experiences of running co-design workshops with participants who have learning disabilities [14], [12]. The co-design sessions were recorded for future analysis as granted by Nottingham Trent University, ethics application 18/19-43V2.

3.1.1 Introduction and demonstration. When conducting co-design workshops with participants who have learning disabilities it is imperative to ensure all participants fully understand the goal of the workshop to improve communication. To ensure this, an experienced facilitator introduced the session by clearly explaining the concept of mental wellbeing tangible interfaces.

In the co-design processes the adage "show me don't tell me" [15] is often used resulting in previously developed prototypes such as the cube shown in Figure 1 being demonstrated to the participants, as existing prototypes can help the participants to understand the feasibility of developing new interfaces. Each prototype embedded similar sensors including 9 Degree of Freedom Inertial Measurement Units (9-DOF IMU) to measure motion, Force Sensitive Resistors (FSR) to measure touch, HR sensors and EDA sensors to measure physiological changes.

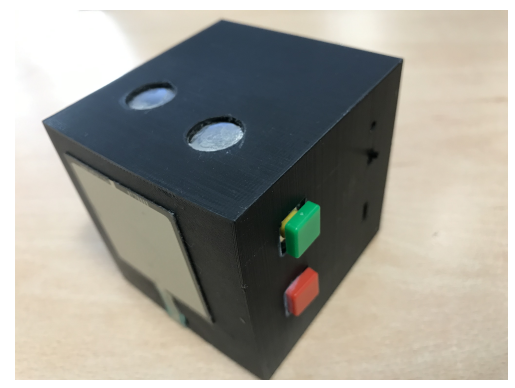

Fig. 1. Original mental wellbeing interface prototype embedding EDA, HR, IMU, FSR and buttons. 
3.1.2 Storyboarding and drawing. The design of the interfaces was explored next. To help compensate for the participants' learning disabilities, real-time storyboarding was completed where prompts were presented to participants to expand upon, promoting communication. During storyboarding, participants were able to discuss their opinions on the existing interfaces previously demonstrated and share their ideas for new interfaces.

In co-design, methods are used to help participants 'say, do and make' [51]. This helps us deepen our engagement with people and strengthens the insights we are able to gather. Using this approach participants were invited to draw their own interfaces using pen and paper to help promote ideas for new interfaces. This enabled the participants to creatively express their design ideas without the need to verbalise, which those with learning disabilities can find challenging.

3.1.3 Real-time 3D printing. The process of showing participants with a learning disability how their design decisions have a direct real world consequence in a rapid and concrete way was developed in an earlier study and replicated here [13]. 3D modelling software was used to demonstrate how the interfaces can be designed and printed to make concrete the relationship between the participants' decisions and the tangible interface produced. The participants voted to 3D print a torus and a cylinder as new interfaces during the workshop due to their simple design, being easy to hold and ideal in which to embed the physiological sensors. Creating the interfaces during the workshops resulted in a deeper and more practical understanding about the participants' experiences [51].

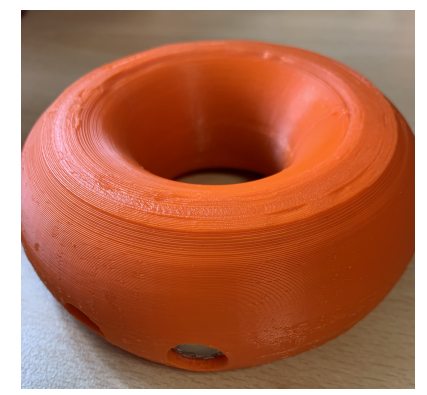

Fig. 2. Torus interface selected to 3D print during the co-design workshop

3.1.4 Prioritising requirement cards. The potential features of tangible interfaces were next explored as it is imperative to understand what features users most require to ensure successful devices are developed. A card based approach was used that enabled participants to physically order the features they believed were most required. This approach was based on the generative research approach [50] to combine participatory exercises with verbal discussion during the creative idea generation phase. Six cards were provided to each participant stating a specific requirement for tangible interfaces including: ease of use, makes me feel better, design, battery life, physical size and understands how I am feeling. Each of the six requirements were explained to the participants by the researcher and experienced facilitator to ensure they fully understood the meaning of the requirements and their role in prioritising the requirement cards.

3.1.5 Interactive electronics. When exploring new technological solutions, providing demonstrations is necessary to ensure all participants understand the functionality and how the technology can be used, thereby improving confidence and communication.

During the session a range of non-invasive, easy to use sensors that could be used in realworld environments [2] were explored through interactive demonstrations, ensuring participants 
understood their functionality by allowing them to test each of the sensors. A HR sensor was first explored where participants were able to place their finger on the sensor and lights would flash at the same rate as their pulse. An EDA sensor was also explained as it functions in a similar way to the HR sensor with participants having to place their fingers on the sensor. A Force Sensitive Resistor (FSR) was demonstrated next, as participants pressed harder on the sensor it caused a haptic motor to vibrate. Finally, a 9-DOF IMU was demonstrated inside a ball; as participants shook the ball it would vibrate. Overall, this method of exploring the sensors promoted participants understanding and enabled them to experience how the sensors will be used in future interfaces.

Varying forms of feedback to act as real-time interventions were then explored giving participants time to reflect and express their feedback between demonstrations. Visual feedback continued to be explored following the use of multicoloured LEDs to demonstrate the HR sensor. Participants were shown multiple examples including one device where different colours represented different emotions. Auditory feedback was also demonstrated where a speaker was used to play calming sounds from nature. Haptic feedback was the last intervention explored; four different feedback patterns were demonstrated to each of the participants who held the vibration motor to experience the different sensations. The exploration of feedback interventions through interactive sessions enabled all participants to experience each of the feedback mechanisms, allowing them to provide personal insights and share their immediate impression of each feedback.

\subsection{Analysis}

After the workshops, the designs, priorities and feedback was collected and analysed. Video and audio recordings of the sessions were also analysed using theory-driven thematic analysis [11]. During thematic analysis, codes are used to describe specific topics from the recordings and then themes are created from the codes to answer the four research questions.

When conducting thematic analysis of the video and audio data, whenever a possible feature to include or exclude was discussed, it was coded. This approach highlighted all of the recommendations suggested by potential users during the co-design workshop and the justification of each feature.

After coding the workshop, similar codes were grouped together to create initial themes. Each theme contains feature suggestions or restrictions answering the initial questions used to create the codes. Each code was then checked within the themes to ensure its suitability, resulting in minor changes to the themes. The thematic analysis process resulted in seven themes created from the codes which were all consistent and represented the feedback from the workshops.

\subsection{Findings}

For each of the seven themes discussed, relevant excerpts from the recordings are provided to highlight the opinions of potential users experiencing mental wellbeing challenges. The complete list of recommendations organised by themes is shown in Table 3.3.7.

3.3.1 Design and Personalisation. When considering the design of the interfaces size was a key factor, it was stated the interfaces should be "not too big and not too small". The size of the existing cube prototype was liked by participants with them stating "like that size" when referring to the size of new interfaces. However, when discussing compromises with size all participants agreed they would prefer slightly larger devices with additional sensors, stating "a big device with everything... would be really handy". Participants also wanted the ability to use it anywhere including at home or at work so a rechargeable battery is necessary.

When exploring the existing prototypes opinions varied as to whether the hard 3D printed shapes or the soft interfaces were preferred with most participants suggesting softer devices for younger 
children and 3D printed interfaces for older children and adults. The colour and personalisation of devices was repeatably mentioned by participants and in particular it was stated the devices should "not be black and white" for children and participants would like the ability for the devices to "change different colours". When asked whether everyone could use a similar device or whether personalised devices would be required, participants suggested the idea of a base interface being developed that can then be customised with cases making the device more personal.

3.3.2 User engagement. When considering potential users of the device, children were suggested multiple times throughout the workshops. It was agreed that younger children aged around 5-8 (middle childhood) would be engaged by softer interfaces stating "these are for the kids" . It was also stated that the larger softer devices may be more appropriate for younger children with less parts to break or chew. The 3D printed interfaces were considered less appropriate for children but would be more engaging for older children and adults to use during everyday life due to their inconspicuous design.

When drawing future interfaces, a novel method to personalise the interfaces by attaching extensions that contain additional sensors or feedback was devised to increase engagement. The addition of extensions would enable the devices to adapt to the user enabling the most beneficial sensors and feedback to be included on an individual basis.

3.3.3 Sensor Inputs. When the device inputs were explored, participants were successfully able to use all of the tested sensors. Participants found the IMU and FSR sensors the easiest and most natural to use. However, not all sensors would be appropriate for children, such as the heart rate sensor, as children would not be able to keep their finger continuously touching the sensor for the device to accurately measure changes in heart rate [74]. It was suggested that physiological sensors should be reserved for the 3D printed interfaces and not the soft interfaces, where children in particular may continuously move their hands preventing accurate readings.

The ability for the interfaces to record messages was a popular request for the soft interfaces. Participants stated they would like to "talk to it about how you feel" and they would "want it to record what is being said ... and play it back". Participants believed the soft toy-like interfaces could act as friends for children where they could express their emotions.

The use of accelerometers to measure motion was well received with participants, finding it useful for the device to recognise how it is being handled such as being bounced or thrown. Accelerometers could be easily embedded within all interfaces, with participants believing the manner in which the devices will be interacted with will be different depending on the user's state of wellbeing. Similarly, force resistive sensors to measure touch could be embedded within both 3D printed and soft interfaces. The ability to measure touch is useful as participants believed stroking the soft interfaces was relaxing as it simulated stroking a pet. The way in which the device was touched was also suggested as a mechanism to indicate wellbeing, with users potentially squeezing the device harder when angry. Participants enjoyed interacting with touch to activate the feedback, such as pressing hard to enable the visual and haptic feedback as they found this method of interaction intuitive. Overall, the touch sensors and accelerometers were preferred by participants as they were simple to interact with and can be embedded within all interfaces for all potential users.

The inclusion of buttons within the interfaces was proposed, with participants liking the buttons to fidget with to potentially improve wellbeing and to initiate feedback. A keyboard input was also suggested, although inclusion of this feature would result in the keys performing the same function as buttons. The majority of the inputs suggested can be implemented using force sensors to measure touch, stroking and squeezing, IMU to measure shaking, HR sensor to measure heart rate and buttons for fidgeting. 
3.3.4 Feedback. When considering outputs, the ability for the devices to make sounds was frequently mentioned. Participants liked the audio feedback playing calming sounds but it was suggested that alternative sounds could also be played to improve mood. Participants believed the ability for interfaces to play calming sounds, music, stories or recorded messages may greatly help improve wellbeing. Guided relaxation was also explained to participants as a potential method to assist relaxation, which participants preferred along with recorded messages. However, a common criticism with the auditory feedback was the quality of the reproduced sound. This could be improved by using a larger speaker, although this would increase the physical size of the device to larger than what participants believed would be comfortable.

Visual feedback was suggested multiple times throughout the workshop, suggestions included "letting people see different shapes and colours", "make it flash either once or twice", "different colours like traffic lights", "lights up bright colours" and "disco lights". When participants designed their own interfaces, the majority included lights activated through either buttons or touch. Visual feedback was most popular choice for inclusion in the children's interfaces where it was suggested that lights should be included throughout the interfaces to make them easy to see with "different shapes and colours of lights". Participants found the inclusion of lights to be vital in making the interfaces more engaging as well as helping them to feel relaxed. Varying combination of lights were trialled with participants preferring light matrices rather than individual LEDs, enabling different patterns to be displayed in addition to varying colours. However, when participants were shown an example where different colours represented different emotions, some were not able to understand the concept that different colours could be used to represent different emotions. This suggests that visual feedback can be used as a distraction technique aiming to improve wellbeing but not to convey information to those with a learning disability.

The possibility of embedding a screen within the tangible interfaces was suggested to show pictures and "sometimes show happy, sometimes show sad faces" dependent on the participant's inferred state of wellbeing. While participants suggested adding a screen, they also suggested that directly showing measures of stress through the feedback may induce additional stress, as has been previously found [38]. To ensure no additional stress is caused, visual feedback should only be used to display varying patterns to aid relaxation.

All participants preferred haptic feedback in comparison to the other feedback mechanisms. Upon first experiencing the haptic feedback, participants stated "I love it" and "it's amazing". When testing different haptic patterns the majority of the participants preferred pattern 1 , which involved long subtle consistent vibrations compared with the second most popular choice, pattern 4, which involved short sharp fast vibrations stating "It's quite soft" and "that's better". It is not unexpected that pattern 1 was most preferred due to its slow relaxing nature, however it was surprising that pattern 4 was the second favoured pattern due to its harsh nature that would not usually be considered relaxing as shown in Figure 3. When comparing haptic feedback patterns, one participant stated "all the rest are harder number four is soft", showing that it may be necessary to personalise haptic patterns on an individual basis. Participants also suggested the haptic feedback patterns should remain slow, potentially slower than the user's heartbeat, to ensure they are relaxing. A potential challenge with haptic feedback is users' becoming accustomed to the sensation, although the continuous variation of patterns 1 and 4 helped to alleviate this issue which users' believing it would help them become more aware of their emotions and help them remain calm. 


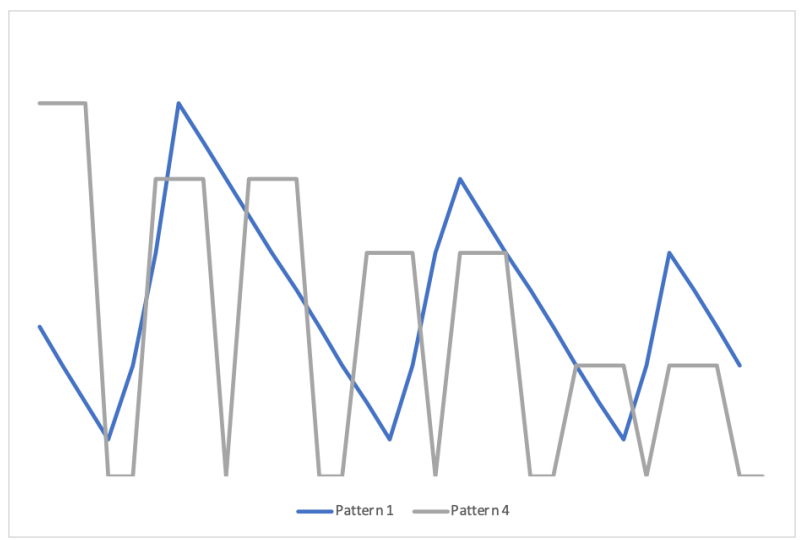

Fig. 3. Vibration amplitude of pattern 1 and pattern 4

Finally, participants made suggestions regarding the ability of the devices to move and change shape. Participants requested the ability for the interfaces to change shape, stretch or "become rounder". Flexible electronics may enable the interfaces to move in this way using motors, although changing shape remains challenging for 3D printed devices.

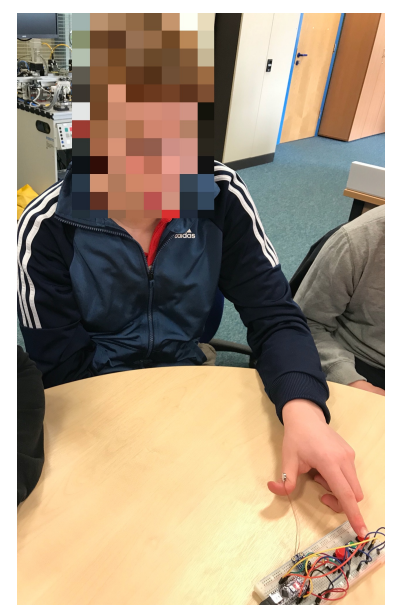

Fig. 4. Participants exploring different haptic patterns during a co-design workshop.

Overall, participants suggested numerous outputs for the design of future devices. Based upon user feedback, haptic feedback is a key intervention that could be played when HR increases and should continue to be explored as all participants found it relaxing and preferred similar vibration patterns. Visual, haptic and auditory feedback can all be embedded within future devices, although shape shifting may be more challenging and the addition of a screen would require careful consideration to ensure it does not induce further stress. Overall, participants believed by including these feedback mechanisms future interfaces could improve their wellbeing in real-time.

3.3.5 Labelling. Methods to label the sensor data were also discussed, as in order to train computational models to infer mental wellbeing a labelled dataset containing the sensor data along 
with the user's state of wellbeing is required. It is necessary to label sensor data at the point of collection as it cannot be labelled offline like other forms of data. On-device labelling simplifies the process of self-reporting by not requiring additional materials, such as questionnaires, and its ease of access promotes frequent labelling.

A variety of buttons, sliding potentiometers and force sensitive resistors were all demonstrated as on-device methods to label wellbeing. All participants preferred buttons stating "I do think they would be better" as they were the easiest to use, in particular using different buttons to represent different emotions. When considering the best methods to represent the buttons, Makaton was suggested which is a language programme that uses signs together with symbols to enable communication [26]. Additionally, different coloured buttons to represent different emotions were explored, such as green and red buttons to represent positive and negative emotions respectively. Using different coloured buttons to label emotional states is easy to use for all participants but the number of labels would be limited to ensure simplicity for those with learning disabilities.

3.3.6 Limitations. During the workshop numerous limitations were discussed but all participants considered it highly important that the interfaces did not appear as a medical device in order to reduce stigma. When a participant previously used medical sensors they stated "I felt awful, I was panicking...the first time I thought I'm not doing this". This makes it vital that any physiological sensors and feedback mechanisms within the interfaces are non-invasive, easy to use and inconspicuous, as to not induce additional stress. As the sensors explored during the workshop were all small and unobtrusive, participants believed they were ideal to monitor real-world mental wellbeing. By developing wellbeing interfaces for the general population, as well as for those experiencing with mental health challenges, it will reduce the associated stigma by ensuring the devices are suitable for all.

When exploring haptic feedback, all participants liked the varying haptic patterns but when initially experiencing the full powered constant haptic motor, it was "too much" for one participant, while another did not like the noise when the motor vibrated against the table. This demonstrates that while haptic feedback was enjoyed and should be explored, the haptic patterns played within the devices can have a major impact on enjoyment and wellbeing. On the other hand, when the haptic motor was embedded within a large soft toy one participant could not feel the motor, showing a careful balance is required to ensure the haptic feedback can be felt within the interfaces but is not overpowering.

Finally, participants stated they would like to have all of the sensors and feedback within one device, however this would drastically decrease battery life and increase the size and cost of the interface. The soft interfaces contain ample space to embed a variety of sensors and feedback However, the 3D printed interfaces are very limited in space especially if they are designed to be small enough to use in the real-world, resulting in only those sensors and feedback preferred by the majority of participants being included.

3.3.7 Communication. During the workshops, lack of communication skills was a theme that appeared throughout. Frequently participants would require prompting when discussing specific topics, for example when explaining which sensors would be most appropriate for use. There was a large variation in the communication skills within the group, with some participants elaborating on their feedback in great detail and others who frequently replied with one-word answers, simply nod or shake their head or always agree with the other participants. These are challenging issues to overcome as some participants may not feel comfortable in expressing their opinions in groups, although the involvement of an experienced facilitator who understood the commonly occurring communication issues for this group, along with the use of interactive activities during the co-design workshops, helped improve communication with all participants. 
Providing initial prototypes for participants to explore aided their understanding of the potential uses for the devices helping them to communicate new ideas and features. The interactive sessions were all received extremely positively. These sessions helped participants gain a clearer understanding of the interfaces and the technologies used within them, helping many to better communicate their ideas and hence provide qualitative data to help answer the research questions.

Drawing new interfaces helped some participants creatively express their ideas that they were not able to verbally communicate such as the sensors and feedback they believed were most important, although other participants struggled to draw an example device. 3D printing during the workshops helped demonstrate how the interfaces are developed and encouraged all participants to consider how different shaped interfaces could be used. This process helped participants make a concrete connection between their design decisions (their drawings) and how this had a direct impact on the outcome (3D printed interface) [13]. Ranking features of the interfaces was slightly more challenging for some participants, although after additional guidance the majority of participants successfully prioritised the features. This helped them express what functionality they believed was most important without needing to verbally communicate. Finally, exploring the sensors and feedback was enjoyed by all and the analysis of the video showed it greatly improved attention and engagement in addition to aiding the understanding of each technology. Demonstrating how the electronics work during the co-design workshop aided participants' understanding of how the devices are developed and helped them make realistic suggestions regarding the design of future tangible interfaces.

p2.5cmp $1.5 \mathrm{cmp} 8 \mathrm{~cm}$

Design and Personalisation Battery life The interfaces must include a rechargeable battery that can power the device throughout the day where it can be used in real-world environments but must also remain small enough to fit within the interfaces.

Colour The interfaces should be developed in a variety of colours enabling them to feel personal. Shape A variety of shapes including 3D printed interfaces and softer interfaces such as children's toys should be developed to cater for all potential users.

User Engagement Children Soft children's toys should be developed that embed the necessary child friendly electronics and interventions such as visual and haptic feedback to improve engagement.

Extensions Develop a fundamental interface that can then be expanded upon to customise the sensors and feedback on an individual basis.

Sensor Inputs Physiological Sensors to measure physiological changes such as HR and EDA should be utilised to help the inference of wellbeing but their use may not be suitable in soft children's interfaces.

Motion Accelerometers to measure motion can easily be embedded within all interfaces and may show how device interactions differ with mood.

Touch The ability to recognise where and how hard the device is touched may help infer wellbeing 
with stroking possibly providing relaxation.

Buttons Buttons to promote fidgeting or to activate feedback should be included to improve individuals' wellbeing.

Microphone It may be possible to infer wellbeing via voice although this imposes privacy issues.

Feedback Sound Calming sound, guided relaxation, stories or voice recordings could be played to aid relaxation.

Haptic Calming haptic feedback patterns can be played when a user is stressed to help improve wellbeing.

Visual Lights can be displayed in various shapes and colours to convey information or act as a distraction to calm users.

Movement Motors make it possible for interfaces to physically move or change shape providing tactile feedback to improve wellbeing.

Labelling Techniques Buttons were the simplest method to label wellbeing in real-time although only a small number of buttons can be embedded within the interfaces limiting the labels.

Representation Different coloured buttons enable users to easily distinguish between the labels, Makaton symbols were also considered but may be more challenging to interpret.

Limitations Size The interfaces need to remain small enough to be portable while also embedding the required sensors and feedback. Individuals may have their own preference to which sensors and feedback function most effectively resulting in personalised devices.

Stigma It is vital the interfaces do not resemble medical devices to make them ubiquitous and reduce stigma as the interfaces should benefit anyone experiencing poor mental wellbeing.

Haptic sensation It is vital haptic feedback can be felt within the interface but it must also not be overpowering to ensure it has a positive impact on wellbeing.

Communication Prototypes The development of initial prototypes aided the understanding of the functionality required within the interfaces, improving communication.

Interactivity Interactive sessions improved engagement and enabled greater communication regarding the design, sensors and feedback for wellbeing interfaces.

\subsection{Requirements}

During the first workshop participants also explored the requirements of tangible wellbeing interfaces, Table 3 shows the order in which five participants prioritised the requirements during the first workshop as one participant was unable to complete the task.

Each requirement, was given a score dependent on the order each participant placed the requirement, where the highest priority was given a score of six and the lowest one. The most prioritised requirement was makes me feel better (22), closely followed by ease of use (21), then battery life (19), size (16), understands how I feel (14) and finally design (13). This shows that the participants all value the feedback the device could provide to make them feel better as the highest priority. However, this would first require the device to understand how the individual is feeling which was second least prioritised feature, possibly showing a lack of understanding of the "understands how I am feeling" requirement. This is not unsurprising as this requirement is the most complex and requires the understanding that a computer model is capable of interpreting emotions. Ease of use was the second highest rated priority showing it to be highly valued amongst all participants. Battery life and size followed, although this greatly varies between participants, with some participants rating them as the highest priority and others rating them as the lowest priority. The lowest overall 
Table 3. Participants' tangible interfaces priorities ordered from highest to lowest priority

\begin{tabular}{|l|l|l|l|l|}
\hline Participant 1 & Participant 2 & Participant 3 & Participant 4 & Participant 5 \\
\hline Ease of use & Battery life & $\begin{array}{l}\text { Makes me feel } \\
\text { better }\end{array}$ & Size & Battery life \\
\hline $\begin{array}{l}\text { Makes me feel } \\
\text { better }\end{array}$ & $\begin{array}{l}\text { Makes me feel } \\
\text { better }\end{array}$ & $\begin{array}{l}\text { Understands } \\
\text { how I am } \\
\text { feeling }\end{array}$ & $\begin{array}{l}\text { Makes me feel } \\
\text { better }\end{array}$ & Ease of use \\
\hline Design & Ease of use & Ease of use & $\begin{array}{l}\text { Understands } \\
\text { how I am } \\
\text { feeling }\end{array}$ & Size \\
\hline $\begin{array}{l}\text { Battery life } \\
\text { Size }\end{array}$ & $\begin{array}{l}\text { Design } \\
\text { how I am } \\
\text { feeling }\end{array}$ & Size & Battery life & Design \\
\hline $\begin{array}{l}\text { Understands } \\
\text { how I am } \\
\text { feeling }\end{array}$ & $\begin{array}{l}\text { Size } \\
\text { Design }\end{array}$ & $\begin{array}{l}\text { Ease of use } \\
\text { how I I am } \\
\text { feeling }\end{array}$ \\
\hline
\end{tabular}

priority was design which was unexpected as participants previously enjoyed exploring the different prototypes suggesting they possibly did not fully understand the implications their design decisions have on the functionality of the resulting devices.

\subsection{Conclusion}

The co-design workshops helped establish the requirements of TUIs such as the inclusion of HR, EDA, force sensors and accelerometers, as well as the preference for haptic feedback to improve wellbeing which can be embedded into future prototypes. Highly important feedback regarding the design of the interfaces was also provided as a range of interfaces should be developed that include soft devices for children and 3D printed shapes which should be portable and not resemble medical devices. Overall, the use of interactive sessions improved participants' ability to understand the interfaces and communicate valuable feedback on the design, relevant inputs and outputs and methods to label the sensor data for training machine learning classifiers.

\section{DESIGN REVISIONS}

Following feedback gained during the co-design workshop a number of prototypes were developed and categorised into soft toys for young children aged 5-8 (middle childhood) as this is when children develop relevant social, emotional and cognitive skills [28] [46] and 3D printed interfaces for older children (8+) and adults. Age is an important factor to consider when developing tangible interfaces for children to ensure they are approachable and engaging, helping to reduce stigma with mental wellbeing tools. Privacy was a key consideration when developing the interfaces. Therefore, the devices do not communicate with any external devices as all data processing and storage is local. Additionally, microphones were not included within the interfaces to capture audio data as the continuous recording of voice to infer wellbeing is highly sensitive and may raise privacy concerns [36]. Speakers were also dismissed due to the requirement for large speakers to play better quality audio.

Initially, the soft children's prototypes were developed. Learning from the feedback from the codesign workshops, physiological sensors were omitted from these interfaces, instead only touch and 
motion interactions would be measured. An FSR was initially explored to measure touch, however the rigid shape of the sensor and limited contact space reduced its potential. Conductive fabrics were then explored as an alternative capacitive sensor to measure resistance. Conductive fabric can be shaped to cover the entirety of the interface enabling all touch interactions to be detected. Using conductive fabric as a capacitive sensor enables the location and pressure of interactions to be measured with the same accuracy as an FSR, while additionally enabling all of the interface's surface to be monitored. To measure motion a 9-DOF IMU continued to be used as its small size and inclusion of an accelerometer, gyroscope and compass provides a large sample of data to monitor interactions. Figure 5 shows the sensors connected to a breadboard as used in the co-design workshops, compared with the sensors placed within the prototype interfaces.

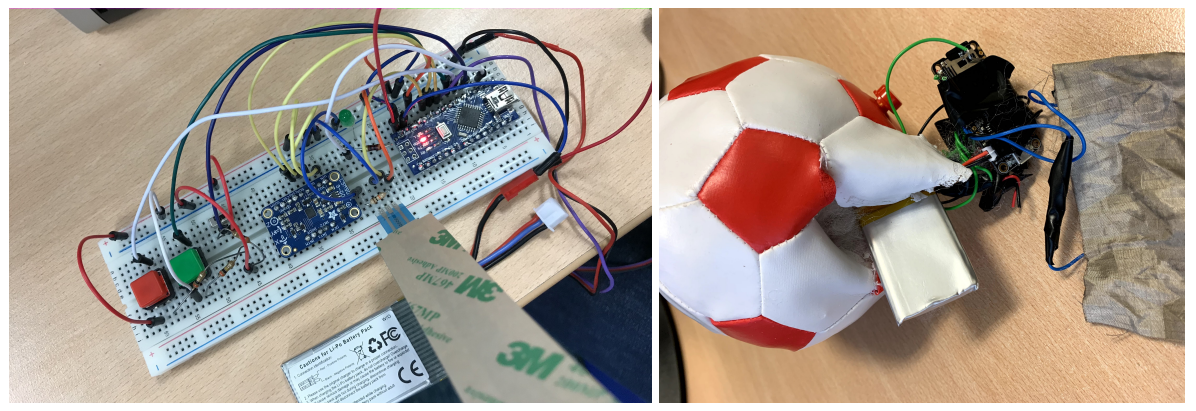

Fig. 5. IMU and FSR connected to microcontroller on a breadboard (left), compared with capacitive sensor and IMU soldered to a microcontroller (right).

Next, the 3D printed interfaces were developed for older children and adults. The physiological sensors including the HR and EDA sensors explored during the co-design workshops performed well and would be beneficial for monitoring wellbeing, however the large size of the EDA sensor was problematic. Conductive materials were explored to build a conductive surface to measure skin conductance. A conductive PLA used to 3D print the interfaces was tested as the possibility of printing a conductive surface would enable the surface of the interface itself to function as an EDA sensor. However, after exploring multiple conductive filaments, none were sufficiently conductive to function as an EDA sensor. It is possible to use other conductive materials as an EDA sensor to measure skin conductance rather than conductive filament, although when using conductive fabric as an EDA sensor the resistance was noticeably lower than the original EDA sensor. Due to the limited nature of conductive materials to function as a reliable EDA sensor, the original EDA sensor in addition to the HR sensor were soldered to the microcontroller creating the electrical components for the 3D printed interfaces as shown in Figure 6. The placement of the physiological sensors within the interfaces was greatly considered to ensure the user's thumb and fingers would be ideally placed to rest on the sensors.

Fidgeting tools were also suggested for the interfaces. When experiencing poor mental wellbeing, people often fidget with objects as fidgeting is a natural response that helps regulate stress [42], [32]. Previous research shows squeezing interactions are preferred by children when angry but boredom was the most prevalent emotion to trigger fidgeting and clicking was preferred when bored [18]. This demonstrates that the fidgeting buttons enjoyed during the co-design workshop are a beneficial addition and should be embedded within mental wellbeing TUIs. 


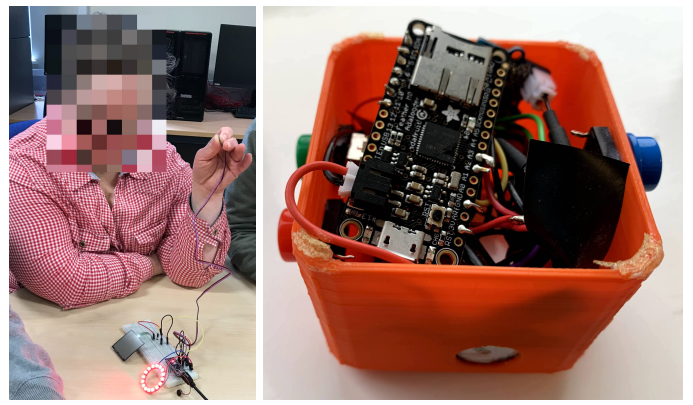

Fig. 6. HR sensor connected to a breadboard used during the co-design workshop (left), compared with soldered sensors within the 3D printed cube (right).

All of the sensors were soldered to an Arduino nano microcontroller due to its small size and wide compatibility with an array of sensors. A battery was also connected with each of the devices providing over 10 hours of continued use in addition to an SD card reader to record all of the data. An example schematic for the 3D printed interfaces is shown in Figure 7.

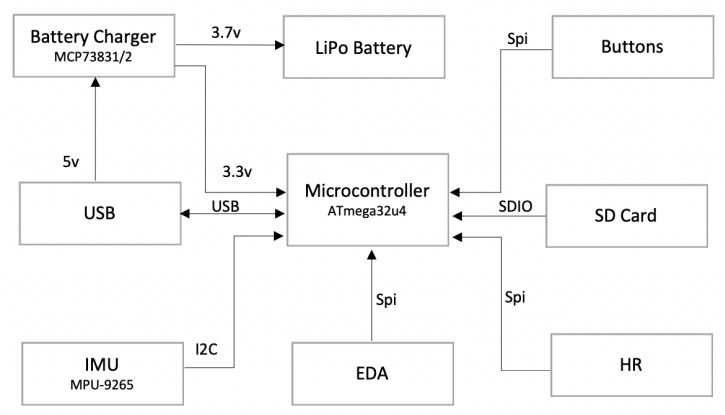

Fig. 7. Schematic showing microcontroller connections with IMU, HR sensor, EDA sensor, SD card and battery.

Overall, six prototypes were developed including both 3D printed shapes and soft interfaces. A soft ball and teddy were designed to cater for all children, while a soft cushion embedded a large skin conductance sensor created from conductive material to enable its performance to be evaluated. The 3D printed interfaces included 2 cubes as these are easy to hold, in addition to the torus shape selected during the workshop. The sensors within the devices vary with each device additionally including one green and one red button to label positive and negative emotions respectively, as shown in Table 4.

\section{DATA COLLECTION}

The data collection approaches were continued to be co-designed during focus groups with the NICER group members and teaching support staff. Initially, it was explained to all participants (via the experienced facilitator) that before a computer model can infer their emotions, sensor data along with self-reported labels were required to train the models. Potential methods to collect a labelled dataset were then explored with the group. Participants agreed with the findings from the collaborative workshops that buttons presented the simplest method of self-reporting and 
Table 4. Developed wellbeing TUIs for real-world sensor data collection

\begin{tabular}{|c|c|c|}
\hline Device & Image & Description \\
\hline Ball & & $\begin{array}{l}\text { A soft ball embedding 9-DOF IMU to mea- } \\
\text { sure motion, force sensors to measure } \\
\text { touch and Multi-coloured LEDs to perform } \\
\text { visual feedback }\end{array}$ \\
\hline $\begin{array}{l}\text { Cube } \\
\text { (touch) }\end{array}$ & & $\begin{array}{l}\text { A 3D printed cube embedding a 9-DOF } \\
\text { IMU, capacitive touch, HR and EDA sen- } \\
\text { sors and haptic feedback }\end{array}$ \\
\hline $\begin{array}{l}\text { Cube (but- } \\
\text { tons) }\end{array}$ & & $\begin{array}{l}\text { A 3D printed cube embedding a 9-DOF } \\
\text { IMU, fidgeting buttons, HR and EDA sen- } \\
\text { sors and haptic feedback }\end{array}$ \\
\hline Teddy & & $\begin{array}{l}\text { A soft teddy embedding a 9-DOF IMU and } \\
\text { capacitive touch sensor and visual feed- } \\
\text { back }\end{array}$ \\
\hline Torus & & $\begin{array}{l}\text { A 3D printed torus embedding a HR, EDA, } \\
\text { 9-DOF IMU and capacitive touch sensor } \\
\text { and haptic feedback }\end{array}$ \\
\hline Cushion & & $\begin{array}{l}\text { A soft cushion embedding a EDA, 9-DOF } \\
\text { IMU and capacitive touch sensors and hap- } \\
\text { tic feedback and visual feedback }\end{array}$ \\
\hline
\end{tabular}

participants preferred the use of different colours to represent different emotions. While some participants suggested three labelling buttons for positive, neutral and negative emotional states, the majority found this confusing and preferred two buttons to represent positive and negative emotions. 
To resolve the lack of on-device labelling mechanisms while ensuring the interfaces are still easy to use, the use of a paper diary was also suggested to additionally record wellbeing, allowing for a larger range of emotional states to be captured and to ensure the on-device labelling techniques are used correctly. Potential emotions and their representations were discussed and co-created, with emojis being favoured by the majority of participants to represent five different emotions including happy, neutral, sad, stressed and frustrated. This approach was led by the experienced facilitator.

\subsection{Method}

Six participants each used one of the tangible interfaces over a two-week period. Participants were instructed to use the red and green buttons to label negative and positive states of wellbeing respectively whenever using the interface. In addition to being given a device to use in their home and college environments, each participant was provided with a diary to record their emojis as shown in Figure 8. 


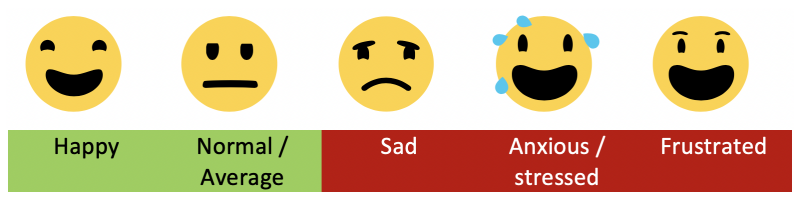

Fig. 8. The emotions used in the diary enabling participants to record additional labels

To ensure the diary was easy to use, five emotions (happy, neutral, sad, stressed and frustrated) were represented using emojis, ensuring emotions from each of the four quadrants from Russell's circumplex [45] were included as shown in Figure 9. Each page of the diary consisted of three sets of emotions enabling labelling each morning, afternoon and evening of the data collection period.

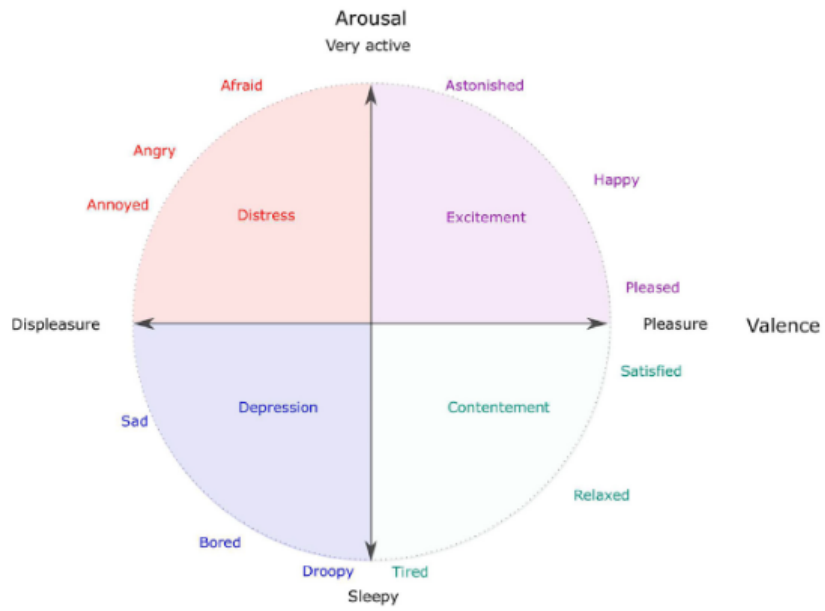

Fig. 9. Russell's circumplex model of affect

\subsection{Findings}

After the two-week data collection period the devices along with the diaries were collected for analysis. One participant had to be immediately excluded due to the unexpected circumstance of their dog chewing the device. The remaining five participants completed their diaries and successfully used the device every day during the two-week period. However, upon evaluation of the diaries and the on-device labels it was shown that three of the five remaining participants were consistently reporting their emotion as happy or neutral throughout the two-week data collection period and never reported negative emotions. One of the participants who recorded negative emotions only recorded two instances of such affect states during the two-week period, while the final participants added text to justify their selection of emotion and subsequently reported the most negative emotions.

When comparing the button labels with the diary entries some anomalies were discovered where the red button had been pressed to record a negative emotion, but a positive emotion was recorded in the diary. This potentially shows the difficulty of using buttons to label data as participants may use the buttons as a fidgeting tool rather than their intended purpose to label the data. The participant who recorded two negative emotions frequently changed their label in a short period of 
time confirming that buttons were potentially purely viewed as methods of interaction rather than a labelling technique. At other times the same participant recorded a label for around 30 seconds then recorded a different label for over a minute showing either their emotion changed frequently, or it took an extended period for the participant to correctly label their wellbeing state. A potential challenge is that when users are stressed they may not pay much attention to correctly labelling their wellbeing states. Overall, the lack of negative emotions during this data collection trial in both the diaries and the on-device labels resulted in a biased dataset which is not sufficient to train machine learning classifiers and demonstrates that participants require further training on how to use the interfaces and labelling method. A potential explanation to the overwhelmingly positive recorded emotions suggested by an experienced teacher of students with learning disabilities is that participants with a learning disability are often keen to please researchers and give positive responses to questions. To address this issue alternative methods have to be devised to gather their affect states.

\section{EVALUATION}

\subsection{Focus Group}

After the data collection trial, another focus group was held where the participants who partook in the data collection study, along with other members of the NICER group, were provided with the opportunity to give feedback both on the tangible interfaces developed and the diary used to provide additional self-reported labels.

During focus groups participants stated that the six tangible interfaces developed thus far are suitable for potential users. The use of the two embedded buttons to label the data was considered appropriate and easy to use. Additional suggestions to improve the devices included changing the colour of the devices and adding a light to make it easier to see which colour button had been pressed, although this could lead to participants selecting a button based upon their colour preference rather than their current wellbeing state. Adding additional buttons to all of the interfaces, that can be pressed at any time to help alleviate stress and used in the manner of fidgeting buttons was also suggested.

Participants stated using the device made them feel happy with the fidgeting buttons, helping them feel calmer. Teachers and end users agreed they liked the shapes of the 3D printed interfaces in addition to liking the ubiquitous nature of the devices with the sensors being embedded within softer objects such as cushions and teddies. Overall, the devices were found to be suitable for their intended purpose of data collection, with some participants finding the fidgeting aspects relaxing even before the interventional feedback had been applied.

The majority of the feedback provided regarded the diary used to record the mental wellbeing of participants. Nearly all participants found the stressed and angry emojis used in the diaries difficult to understand. During a further feedback session with the NICER group, the use photo realistic images of faces [8] was proposed, which may be easier to understand than emojis. Alternatively, it was suggested to change the colours of the emoji faces, where the happy face would be green and the angry face red.

The frequency of the self-reporting was also problematic as the diary only allowed participants to record their emotions over long periods of time, such as one label for the morning or afternoon. However, when stress or anger was experienced it was only for short or transient period, but as the individual felt happy for majority of the time frame it resulted in positive emotions being recorded even though negative emotions were also experienced. To resolve this issue it was suggested that the diaries could ask for participants' mental wellbeing states to be recorded during the past hour as this should increase the frequency of emotional variation. Users could also be instructed to use 
the device whenever they feel stressed or angry as these emotions don't usually last very long, to increase the frequency of data collection related to poor mental wellbeing states being collected allowing for the successful training of the machine learning classifiers.

Additionally, it was suggested that parents or carers could help provide insights into the mental wellbeing states of participants by completing their own questionnaires to expertly label participant affect states, or justifying each emotion reported. However, the presence of a paid carer may pressure users into responding more positively and if parents and carers only complete questionnaires infrequently they will not capture the transient periods of time where users may experience negative emotions. Further training will be required to ensure that all participants with a learning disability understand each emotion to improve the accuracy of the data collection as there is the need to first train them in understanding and recognising their different emotions before going onto record these as labels.

Following feedback from the evaluation two new diaries were created both removing the previous time frames. The first used photo realistic images of a person expressing five emotions: happy, calm, sad, shocked and angry. The second diary continued using emojis but rather than emojis expressing specific emotions it displayed five emojis that scaled from happy to sad with the colour gradually changing from green to red based on the Self-Assessment Manikin scale [23]. Furthermore, based on feedback from the focus group, in future data collection trials all participants will be trained to use their diaries more frequently, in particular when they experience a change in their emotions to aid the collection of sensor data associated with varying emotional states.

Furthermore, additional 3D printed interfaces were developed following the feedback gained. A smaller cuboid containing only motion and a capacitive touch sensor, a sphere containing motion, HR and EDA sensors and a pebble shaped device containing motion, HR and EDA sensors were developed (in addition to the previously developed devices). These devices were designed to be easier to use with the sphere containing sleeves to place fingers within, making it easier to touch the HR and EDA sensors. The pebble was designed to ensure that when a user holds it in their hand, their thumb will rest on the HR sensor and their palm will touch the EDA sensor. Overall, the feedback from the focus group helped to refine the co-design of the devices themselves, as well as the methods of labelling data which have similarly been co-designed with end users and their teachers/carers.

\subsection{Revised Data Collection}

Before commencing a second data collection trial to collect additional real-world labelled sensory data, the two new diary styles as shown in Figure 10 were shown to participants based upon feedback during the evaluation. The new diaries continued to display five emotions within both the photo realistic diary and the revised emoji diary. After exploring both designs the majority of participants preferred the photo realistic diary and hence this was used for labelling all future data. 


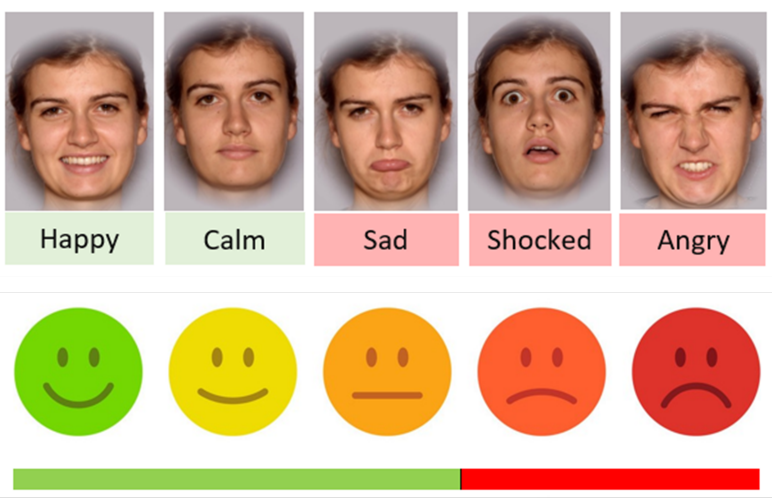

Fig. 10. Updated data labelling diaries using photo realistic images (top) and five point emoji scale (bottom)

Six participants were then asked to partake in a second data collection trial with the interfaces being selected by the participants themselves. Additional training on how to use the devices was then performed. Each of the emotional states were clearly explained to users by the experienced facilitator as there was previously difficulty establishing the difference between the stress and frustration states. These were subsequently changed to shocked and angry emotions which participants found easier to understand. Participants were informed they were to use the devices more frequently during this data collection period, keeping the device with them for as much time as possible. Participants were also instructed to frequently label their wellbeing states, especially when their emotion might change to ensure a wide range of labels connected to different affect states are collected as this was an issue with the previous data collection trial. Once the participants understood the requirement for increased labelling frequency they then selected a device to use with the Torus, Sphere, Pebble, two cubes and Cushion being selected.

After participants used the devices for one week, the interfaces were collected so that the data could be analysed and used for computational analysis. The number of positive and negative samples recorded from each of the interfaces is shown in Table 5. The data from four of the interfaces (users $1,2,36$ ) show a range of positive and negative emotions have been recorded over a period of several days. The data from another device (user 4) only recorded happy data similar to the previous data collection trial, although this could possibly be because the participant only experienced happiness during the data collection period. The final device (user 5) was not used often (only twice), resulting in insufficient data to train a machine learning model.

Table 5. Comparison of positive and negative real-world emotional state samples for all 6 users.

\begin{tabular}{|lll|}
\hline & $\begin{array}{l}\text { Positive } \\
\text { samples }\end{array}$ & $\begin{array}{l}\text { Negative } \\
\text { samples }\end{array}$ \\
\hline User 1 & 14184 & 12568 \\
\hline User 2 & 796 & 15874 \\
\hline User 3 & 1329 & 9438 \\
\hline User 4 & 32586 & 33 \\
\hline User 5 & 706 & 20 \\
\hline User 6 & 12568 & 15184 \\
\hline
\end{tabular}


The on-device labels were used to explore the data as participants often forgot to complete the diary due to the on-device labelling buttons being more convenient. Also, when using the diaries participants regularly used only two emotions, one to represent negative emotions and one to represent positive emotions matching the labelling buttons.

When exploring the EDA data from users 1, 2, 3 and 6 Figure 11 shows the widest distribution for both positive and negative states of wellbeing is similar but when experiencing stress there is an secondary wide distribution. Similarly, when exploring HRV both states share the widest distribution but the distribution is much wider when experiencing negative emotions and the median HRV is higher when experiencing positive emotions. This data demonstrates that the real-world physiological data collected from the tangible interfaces behaves as would be expected with EDA decreasing and HRV increasing when experiencing poor wellbeing.

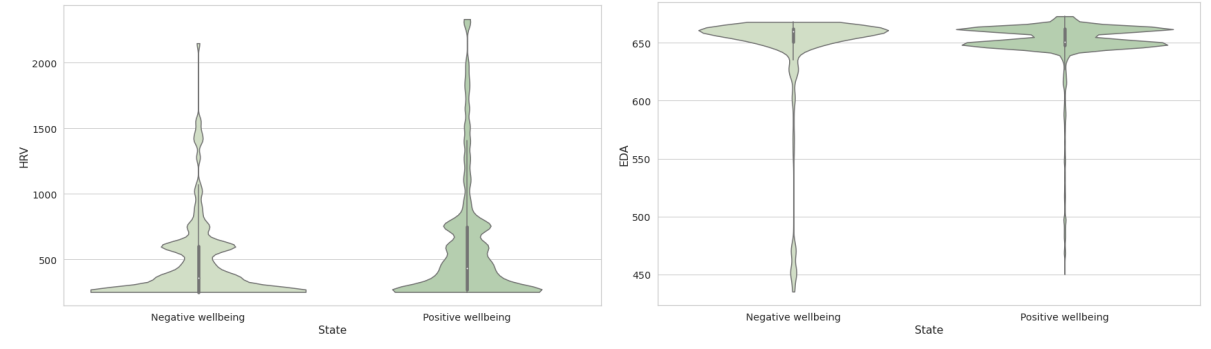

Fig. 11. Comparison of real-world HRV data (left) and EDA data (right) collected using the tangible interfaces by 3 participants.

The additional training shows improved data collection results with a much wider range of emotions recorded for most participants, although issues such as biased data and insufficient data from participants were still encountered. This work paves the way for large scale data collection trials in the future. When conducting wellbeing data collection trials with participants who have learning disabilities, it is vital to first ensure they have a full understanding of how to use the interfaces to record their emotions, as well as encouraging the frequent labelling of different emotions to ensure the data collection is successful.

\section{DISCUSSION}

This co-design study has explored the design, development and technologies of TUIs to monitor real-world mental wellbeing in addition to the methods of labelling mental wellbeing data to train machine learning models. A co-design methodology was adopted based on previous research with participants whose mental wellbeing can often be diagnostically overshadowed and who commonly have difficulty in expressing their emotions. The co-design approach adopted addressed the limitations experienced by people with a learning disabilities (e.g., communication, working memory), enabling them to participate more effectively. This approach takes a practical stance in guiding how co-design methods can be made to work in realistic design settings and adjusted to the needs of participants who may experience learning disabilities.

Co-designing for people with severe, profound and complex learning and physical disabilities has shown additional challenges as not all tasks were successfully completed - such as drawing a new interface design. Significant communication challenges were also present. However, the majority of the tasks were successful, particularly the interactive activities within the co-design workshop, involving the 3D printing of new interface designs to immediately show participants the results of their design decisions and exploring the sensors and feedback mechanisms. These interactive 
activities helped maintain engagement, improved understanding of new concepts and improved communication within the workshops. By combining interactive sessions with storyboarding to gain feedback, it has been possible to gain valuable insights aiding the design and development of future wellbeing interfaces as shown in Table 6.

Table 6. Development insights from co-design workshops.

\begin{tabular}{|c|l|}
\hline Category & Insight \\
\hline Design & $\begin{array}{l}\text { Different devices should be developed for different } \\
\text { age ranges with soft devices for young children } \\
\text { and 3D printed interfaces for older children and } \\
\text { adults. All devices should remain inconspicuous } \\
\text { and not appear as medical devices to help reduce } \\
\text { stigma. }\end{array}$ \\
\hline Sensors & $\begin{array}{l}\text { Touch and motion sensors should be included } \\
\text { within all devices due to their simplicity, with } \\
\text { physiological sensors reserved for 3D printed in- } \\
\text { terfaces. }\end{array}$ \\
\hline Feedback & $\begin{array}{l}\text { Slow haptic feedback should be used within all } \\
\text { interfaces to help relax users. Similarly, fidgeting } \\
\text { buttons were found relaxing and should be incor- } \\
\text { porated into 3D printed interfaces. While visual } \\
\text { feedback was enjoyed, it is challenging to use it to } \\
\text { convey information such as emotional wellbeing. }\end{array}$ \\
\hline Labelling & $\begin{array}{l}\text { On-device labelling buttons simplifies labelling } \\
\text { process but the number of labels is limited to pre- } \\
\text { vent users becoming overwhelmed. Self-report di- } \\
\text { aries using photo realistic images should be used } \\
\text { to collect additional labels. }\end{array}$ \\
\hline
\end{tabular}

A range of designs, sensors, feedback mechanisms and labelling approaches were all explored using co-design approaches that have been adapted to address the limitations faced by participants with learning disabilities. The results from this study show there is no one-size-fits-all solution to the design of mental wellbeing interfaces for this end user population. Instead, a range of devices must be developed encompassing various sensors and feedback to act as interventions. Following the co-design workshops, a range of new interfaces have been developed including 3D printed cubes and soft toys. The interfaces developed are suitable for use by those with learning disabilities to collect labelled wellbeing sensor data and, in the future, to automatically infer mental wellbeing.

During the workshops the experienced facilitator worked with the participants to develop an understanding that in order for the interfaces to automatically infer their wellbeing, a computational model must first be trained using data collected from the devices. The collection of real-world data using the interfaces was vital as previous work mostly explores the use of controlled experimental datasets to infer wellbeing, which may not perform accurately when used in real-world domains.

The process of collecting real-world labelled data was similarly co-designed through focus group sessions. Two methods to label the data were co-designed; the labelling buttons embedded within the interface were selected during the workshops due to their simplicity, while the self-report diaries were selected after a series of focus groups exploring different methods of representing 
emotions. The initial data collection trial demonstrated the challenges of collecting a real-world labelled dataset as the majority of the participants only recorded positive emotions during the initial two-week data collection period. After re-evaluating the data collection method, additional training was provided to users (via experienced facilitator) and by using on-device labelling as well as updated self-reporting diaries, frequent accurate labelling was successfully completed during the second data collection trial. This demonstrates that when developing interfaces to collect data from participants with learning disabilities the entire process must be co-designed to ensure suitable devices are developed, correct labels are recorded and sufficient data is collected. This is very much an iterative process.

In the future, additional data collection trials will be conducted where the labelled data can be used to train machine learning classification models and enable the automatic inference of mental wellbeing. Once mental wellbeing can be inferred automatically it presents the opportunity for the feedback explored during the co-design workshops to be applied, aiming to use such feedback to automatically improve mental wellbeing states in real-time.

\section{CONCLUSION}

Classifying mental wellbeing is a challenging proposition but with recent advances in machine learning and sensors it is now more achievable than ever before. The automatic inference of mental wellbeing would be extremely valuable for individuals with learning disabilities as their wellbeing is often diagnostically overshadowed and they can find it challenging to express their emotions. TUIs enable the necessary microprocessor and sensors required to monitor mental wellbeing to be embedded, in addition to providing feedback mechanisms that may help improve mental wellbeing in real-time.

Inclusive co-design workshops and focus groups have been conducted to rethink the user design approach of wellbeing TUIs. Qualitative data from these sessions has been analysed using thematic analysis. Adjustments to traditional co-design techniques included demonstrations, real-time 3D printing, prioritising cards and interactive electronics to enable successful and practical co-design with people with learning disabilities. In particular, 3D printing of new interfaces improved engagement, ensured participants understood the discussed technologies and demonstrated how their design decisions influenced the final designs. Various designs, sensors and feedback mechanisms were explored during the workshops resulting in many recommendations and a range of new interfaces being developed.

An initial data collection trial to collect a real-world labelled dataset demonstrated common issues, such as mislabelling and infrequent use. These challenges were further explored during a focus group and resolved before a second data collection trial commenced. The data collected from the second trial demonstrated more frequent labelling of varying emotions. In the future, a larger data collection trial can be conducted using the developed interfaces and labelling techniques, which can then be used to train machine learning classifiers to infer real-world mental wellbeing.

Overall, the participatory process has enabled the successful design and development of mental wellbeing TUIs for people with a range of learning disabilities. The developed interfaces have also been used to collect a real-world labelled emotion dataset. In the future, data collected from these devices could be used to train machine learning classifiers and, using edge computing, the model could be run 'on-device' to automatically infer mental wellbeing and apply real-time feedback.

\section{REFERENCES}

[1] Nouf Alajmi, Eiman Kanjo, Nour El Mawass, and Alan Chamberlain. 2013. Shopmobia: An Emotion-Based Shop Rating System. In 2013 Humaine Association Conference on Affective Computing and Intelligent Interaction. IEEE, 745-750. 
[2] Miquel Alfaras, William Primett, Muhammad Umair, Charles Windlin, Pavel Karpashevich, Niaz Chalabianloo, Dionne Bowie, Corina Sas, Pedro Sanches, Kristina Höök, Cem Ersoy, and Hugo Gamboa. 2020. Biosensing and actuationplatforms coupling body input-output modalities for affective technologies. Sensors (Switzerland) (2020). https: //doi.org/10.3390/s20215968

[3] Miquel Alfaras, Vasiliki Tsaknaki, Pedro Sanches, Charles Windlin, Muhammad Umair, Corina Sas, and Kristina Höök. 2020. From Biodata to Somadata. In Conference on Human Factors in Computing Systems - Proceedings. https: //doi.org/10.1145/3313831.3376684

[4] Salma Alhagry, Aly Aly, and Reda A. 2017. Emotion Recognition based on EEG using LSTM Recurrent Neural Network. International fournal of Advanced Computer Science and Applications 8, 10 (2017). https://doi.org/10.14569/ijacsa.2017. 081046

[5] Brenna D. Argall and Aude G. Billard. 2010. A survey of Tactile HumanRobot Interactions. https://doi.org/10.1016/j. robot.2010.07.002

[6] Ruben T Azevedo, Nell Bennett, Andreas Bilicki, Jack Hooper, Fotini Markopoulou, and Manos Tsakiris. 2017. The calming effect of a new wearable device during the anticipation of public speech. Sci Rep 7, 1 (2017), 2285.

[7] Madeline Balaam, Geraldine Fitzpatrick, Judith Good, and Rosemary Luckin. 2009. Exploring Affective Technologies for the Classroom with the Subtle Stone. Proceedings of the 28th international conference on Human factors in computing systems - CHI '10 (2009), 1623.

[8] Paul D. Barrows and Shirley A. Thomas. 2018. Assessment of mood in aphasia following stroke: validation of the Dynamic Visual Analogue Mood Scales (D-VAMS). Clinical Rehabilitation 32, 1 (jan 2018), 94-102. https: //doi.org/10.1177/0269215517714590

[9] Thomas Binder, Giorgio De Michelis, Pelle Ehn, Giulio Jacucci, Per Linde, and Ina Wagner. 2019. Participation in Design Things. In Design Things. https://doi.org/10.7551/mitpress/8262.003.0011

[10] Patricia J. Bota, Chen Wang, Ana L.N. Fred, and Hugo Placido Da Silva. 2019. A Review, Current Challenges, and Future Possibilities on Emotion Recognition Using Machine Learning and Physiological Signals. https://doi.org/10 1109/ACCESS.2019.2944001

[11] Virginia Braun and Victoria Clarke. 2006. Using thematic analysis in psychology. Qualitative Research in Psychology (2006). https://doi.org/10.1191/1478088706qp063oa

[12] D Brown, R Kettley, and S Kettley. 2016. Interpersonal Process Recall - a method for reflecting on co-design experiences. In Method Stories Workshop, ACM CHI 2016. https://chimethodstories.wordpress.com/

[13] David J. Brown, Steven Battersby, Penny Standen, Nicola Anderton, Matthew Harrison, and David J. Brown. 2005 Inclusive design for disability: A prototype switch for use in virtual learning environments. International fournal on Disability and Human Development (2005). https://doi.org/10.1515/IJDHD.2005.4.2.103

[14] David J. Brown, David McHugh, Penny Standen, Lindsay Evett, Nick Shopland, and Steven Battersby. 2011. Designing location-based learning experiences for people with intellectual disabilities and additional sensory impairments. Computers and Education (2011). https://doi.org/10.1016/j.compedu.2010.04.014

[15] Ingrid Burkett. 2016. An Introduction to Co-design. Technical Report. http://www.csi.edu.au/

[16] Yekta Said Can, Bert Arnrich, and Cem Ersoy. 2019. Stress detection in daily life scenarios using smart phones and wearable sensors: A survey. Journal of Biomedical Informatics (2019). https://doi.org/10.1016/j.jbi.2019.103139

[17] Nair Chaitanya M., Sharmila Jayakkumar, Edwin Chong, and C.H. Yeow. 2017. A wearable, EEG-based massage headband for anxiety alleviation. In 2017 39th Annual International Conference of the IEEE Engineering in Medicine and Biology Society (EMBC), Vol. 2017. IEEE, 3557-3560. https://doi.org/10.1109/EMBC.2017.8037625

[18] Suzanne B. Da Câmara, Rakshit Agrawal, and Katherine Isbister. 2018. Identifying children's fidget object preferences: Toward exploring the impacts of fidgeting and fidget-friendly tangibles. In DIS 2018 - Proceedings of the 2018 Designing Interactive Systems Conference. https://doi.org/10.1145/3196709.3196790

[19] Melissa Dawe. 2007. "let me show you what i want": Engaging individuals with cognitive disabilities and their families in design. In Conference on Human Factors in Computing Systems - Proceedings. https://doi.org/10.1145/1240866.1240976

[20] Bo Edvardsson, Thomas Meiren, Adrienne Schäfer, and Lars Witell. 2013. Having a strategy for new service development - does it really matter? fournal of Service Management (2013). https://doi.org/10.1108/09564231311304170

[21] Peter Francis, Sandrine Balbo, and Lucy Firth. 2009. Towards co-design with users who have autism spectrum disorders. Universal Access in the Information Society (2009). https://doi.org/10.1007/s10209-008-0143-y

[22] Carolina Fuentes, Iyubanit Rodríguez, and Valeria Herskovic. 2015. EmoBall: A study on a tangible interface to selfreport emotional information considering digital competences. In Lecture Notes in Computer Science (including subseries Lecture Notes in Artificial Intelligence and Lecture Notes in Bioinformatics). https://doi.org/10.1007/978-3-319-26508-7_19

[23] B. Geethanjali, K. Adalarasu, A. Hemapraba, S. Pravin Kumar, and R. Rajasekeran. 2017. Emotion analysis using sam (Self-assessment manikin) scale. Biomedical Research (India) (2017).

[24] Alison Giraud-Saunders. 2011. Mental health in people with learning disabilities. 
[25] James J Gross and R Thompson. 2007. Emotion regulation: Conceptual foundations. In f. J. Gross (Eds.) Handbook of Emotion Regulation.

[26] Nicola Grove and Margaret Walker. 1990. The Makaton Vocabulary: Using Manual Signs and Graphic Symbols to Develop Interpersonal Communication. Augmentative and Alternative Communication (1990). https://doi.org/10.1080/ 07434619012331275284

[27] Frode Guribye and Tor Gjøsæter. 2018. Tangible Interaction in the Dentist Office. In Proceedings of the Twelfth International Conference on Tangible, Embedded, and Embodied Interaction - TEI '18. ACM Press, New York, New York, USA, 123-130. https://doi.org/10.1145/3173225.3173287

[28] Gordon T. Harold and Dale F. Hay. 2005. Normal development in middle childhood. Psychiatry 4, 6 (jun 2005), 3-5. https://doi.org/10.1383/psyt.4.6.3.66355

[29] J.A. Healey and R.W. Picard. 2005. Detecting Stress During Real-World Driving Tasks Using Physiological Sensors. IEEE Transactions on Intelligent Transportation Systems 6, 2 (jun 2005), 156-166.

[30] Stefan Holmlid. 2009. Participative, co-operative, emancipatory: From participatory design to service design. First Nordic Conference on Service Design and Service Innovation (2009).

[31] Kristina Höök, Martin P. Jonsson, Anna Ståhl, and Johanna Mercurio. 2016. Somaesthetic Appreciation design. In Conference on Human Factors in Computing Systems - Proceedings. https://doi.org/10.1145/2858036.2858583

[32] B. F. Hudson, J. Ogden, and M. S. Whiteley. 2015. Randomized controlled trial to compare the effect of simple distraction interventions on pain and anxiety experienced during conscious surgery. European fournal of Pain (United Kingdom) (2015).

[33] Eiman Kanjo, Eman M.G. Younis, and Chee Siang Ang. 2019. Deep learning analysis of mobile physiological, environmental and location sensor data for emotion detection. Information Fusion 49 (sep 2019), 46-56.

[34] Chelsea Kelling, Daniella Pitaro, and Jussi Rantala. 2016. Good vibes. In Proceedings of the 20th International Academic Mindtrek Conference on - AcademicMindtrek '16. ACM Press, New York, New York, USA, 130-136.

[35] George Poonkhin Khut. 2016. Designing biofeedback artworks for relaxation. In Conference on Human Factors in Computing Systems - Proceedings. https://doi.org/10.1145/2851581.2891089

[36] Jacob Leon Kröger, Otto Hans Martin Lutz, and Philip Raschke. 2020. Privacy implications of voice and speech analysis - information disclosure by inference. In IFIP Advances in Information and Communication Technology. https://doi.org/10.1007/978-3-030-42504-3_16

[37] Chih Lung Lin, Pei Sheng Gau, Kuan Jung Lai, Yu Kaun Chu, and Chien Hsu Chen. 2009. Emotion caster: Tangible emotion sharing device and multimedia display platform for intuitive interactions. In Digest of Technical Papers - IEEE International Conference on Consumer Electronics. https://doi.org/10.1109/ISCE.2009.5156954

[38] Diana MacLean, Asta Roseway, and Mary Czerwinski. 2013. MoodWings. In Proceedings of the 6th International Conference on PErvasive Technologies Related to Assistive Environments - PETRA '13. ACM Press, New York, New York, USA, 1-8. https://doi.org/10.1145/2504335.2504406

[39] Hector P. Martinez, Yoshua Bengio, and Georgios Yannakakis. 2013. Learning deep physiological models of affect. IEEE Computational Intelligence Magazine 8, 2 (2013), 20-33.

[40] Mark Matthews and Gavin Doherty. 2011. In the Mood: Engaging Teenagers in Psychotherapy Using Mobile Phones. Proceedings of the 2011 annual conference on Human factors in computing systems - CHI '11 (2011).

[41] Pardis Miri, Robert Flory, Andero Uusberg, Heather Culbertson, Richard H. Harvey, Agata Kelman, Davis Erik Peper, James J. Gross, Katherine Isbister, and Keith Marzullo. 2020. PIV: Placement, pattern, and personalization of an inconspicuous vibrotactile breathing pacer. ACM Transactions on Computer-Human Interaction (2020). https: //doi.org/10.1145/3365107

[42] Changiz Mohiyeddini and Stuart Semple. 2013. Displacement behaviour regulates the experience of stress in men. Stress (2013).

[43] Karin Niemantsverdriet and Maarten Versteeg. 2016. Interactive Jewellery as Memory Cue. In Proceedings of the TEI '16: Tenth International Conference on Tangible, Embedded, and Embodied Interaction - TEI '16. ACM Press, New York, New York, USA, 532-538. https://doi.org/10.1145/2839462.2856524

[44] Ana Paiva, Marco Costa, Ricardo Chaves, Moisés Piedade, Dário Mourão, Daniel Sobral, Kristina Höök, Gerd Andersson, and Adrian Bullock. 2003. SenToy: An affective sympathetic interface. International fournal of Human Computer Studies (2003). https://doi.org/10.1016/S1071-5819(03)00048-X

[45] James A. Russell. 1980. A circumplex model of affect. Journal of Personality and Social Psychology 39, 6 (dec 1980), 1161-1178. https://doi.org/10.1037/h0077714

[46] Carolyn Saarni. 2011. Emotional development in childhood. Encyclopedia on Early Childhood Develpment (2011).

[47] Pedro Sanches, Kristina Höök, Elsa Vaara, Claus Weymann, Markus Bylund, Pedro Ferreira, Nathalie Peira, and Marie Sjölinder. 2010. Mind the body! Designing a mobile stress management application encouraging personal reflection. In DIS 2010 - Proceedings of the 8th ACM Conference on Designing Interactive Systems. https://doi.org/10.1145/1858171 1858182 
[48] Pedro Sanches, Axel Janson, Pavel Karpashevich, Camille Nadal, Chengcheng Qu, Claudia Daudén Roquet, Muhammad Umair, Charles Windlin, Gavin Doherty, Kristina Höök, and Corina Sas. 2019. HCI and Affective Health Taking stock of a decade of studies and charting future research directions. In Conference on Human Factors in Computing Systems Proceedings. Association for Computing Machinery, 17. https://doi.org/10.1145/3290605.3300475

[49] E Sanders. 2006. Design Research in 2006. Design Research Society (2006).

[50] E. B.-N. Sanders. 2000. Generative Tools for Co-designing. In Collaborative Design. Springer London, 3-12. https: //doi.org/10.1007/978-1-4471-0779-8_1

[51] Elizabeth B. N. Sanders and Uday Dandavate. 1999. Design for Experiencing: New tools. In First International Conference on Design and Emotion.

[52] Liz Sanders. 2008. An evolving map of design practice and design research. Interactions (2008). https://doi.org/10. $1145 / 1409040.1409043$

[53] Martha Patricia Sarmiento-Pelayo. 2015. Co-design: A central approach to the inclusion of people with disabilities. Revista Facultad de Medicina (2015). https://doi.org/10.15446/revfacmed.v63n3sup.49345

[54] Federico Sarzotti. 2018. Self-Monitoring of Emotions and Mood Using a Tangible Approach. Computers 7, 1 (jan 2018), 7.

[55] Johannes Schumm, Eth Zurich, Ulrike Ehlert, Cornelia Setz, Bert Arnrich, Roberto La Marca, and Gerhard Tröster. 2010. Discriminating stress from cognitive load using a wearable EDA device. Discriminating Stress From Cognitive Load Using a Wearable EDA Device. IEEE Transactions on Information Technology in Biomedicine 14, 2 (2010).

[56] Nandita Sharma and Tom Gedeon. 2012. Objective measures, sensors and computational techniques for stress recognition and classification: A survey. Computer Methods and Programs in Biomedicine 108, 3 (dec 2012), 1287-1301.

[57] Takanori Shibata and Kazuyoshi Wada. 2011. Robot Therapy: A New Approach for Mental Healthcare of the Elderly A Mini-Review. Gerontology 57, 4 (2011), 378-386. https://doi.org/10.1159/000319015

[58] Lin Shu, Jinyan Xie, Mingyue Yang, Ziyi Li, Zhenqi Li, Dan Liao, Xiangmin Xu, and Xinyi Yang. 2018. A review of emotion recognition using physiological signals. https://doi.org/10.3390/s18072074

[59] Laurianne Sitbon and Shanjana Farhin. 2017. Co-designing interactive applications with adults with intellectual disability: A case study. In ACM International Conference Proceeding Series. https://doi.org/10.1145/3152771.3156163

[60] Iouliia Skliarova and Valery Sklyarov. 2019. Hardware/software co-design. In Lecture Notes in Electrical Engineering. https://doi.org/10.1007/978-3-030-20721-2_6

[61] Katta Spiel. 2020. SIGCHI outstanding dissertation award: Evaluating experiences of autistic children with technologies in co-design. In Conference on Human Factors in Computing Systems - Proceedings. Association for Computing Machinery, New York, NY, USA, 1-4. https://doi.org/10.1145/3334480.3386151

[62] Katta Spiel, Emeline Brulé, Christopher Frauenberger, Gilles Bailly, and Geraldine Fitzpatrick. 2018. Micro-ethics for participatory design with marginalised children. https://doi.org/10.1145/3210586.3210603

[63] Marc Steen. 2013. Co-design as a process of joint inquiry and imagination. Design Issues (2013). https://doi.org/10. 1162/DESI_a_00207

[64] Marc Steen, Menno Manschot, and Nicole de Koning. 2011. Benefits of co-design in service design projects. International fournal of Design (2011).

[65] B. Ullmer and H. Ishii. 2000. Emerging frameworks for tangible user interfaces. IBM Systems fournal (2000). https: //doi.org/10.1147/sj.393.0915

[66] Muhammad Umair, Muhammad Hamza Latif, and Corina Sas. 2018. Dynamic displays at wrist for real time visualization of affective data. In DIS 2018 - Companion Publication of the 2018 Designing Interactive Systems Conference. https: //doi.org/10.1145/3197391.3205436

[67] Terumi Umematsu, Akane Sano, Sara Taylor, and Rosalind W. Picard. 2019. Improving Students' Daily Life Stress Forecasting using LSTM Neural Networks. Institute of Electrical and Electronics Engineers (IEEE), 1-4.

[68] John Vines, Rachel Clarke, Peter Wright, John McCarthy, and Patrick Olivier. 2013. Configuring participation: On how we involve people in design. In Conference on Human Factors in Computing Systems - Proceedings. https: //doi.org/10.1145/2470654.2470716

[69] Froukje Sleeswijk Visser. 2009. Bringing the everyday life of people into design. https://doi.org/Isbn978-90-9024244-6

[70] Eric von Hippel. 1994. "Sticky Information" and the Locus of Problem Solving: Implications for Innovation. Management Science (1994). https://doi.org/10.1287/mnsc.40.4.429

[71] Joseph Wherton, Paul Sugarhood, Rob Procter, Sue Hinder, and Trisha Greenhalgh. 2015. Co-production in practice: How people with assisted living needs can help design and evolve technologies and services. Implementation Science (2015). https://doi.org/10.1186/s13012-015-0271-8

[72] Charles Windlin. 2020. Designing with the body: Addressing emotion regulation and expression. In DIS 2020 Companion Companion Publication of the 2020 ACM Designing Interactive Systems Conference. Association for Computing Machinery, Inc, New York, NY, USA, 557-562. https://doi.org/10.1145/3393914.3395835 
[73] Kieran Woodward, Eiman Kanjo, David Brown, and T.M. McGinnity. 2020. On-Device Transfer Learning for Personalising Psychological Stress Modelling Using a Convolutional Neural Network. In On-device Intelligence Workshop, MLSys, Austin, Texas.

[74] Kieran Woodward, Eiman Kanjo, David Brown, and T. M. McGinnity. 2019. AI- Powered Tangible Interfaces to Transform Children's Mental Well-being. In The 5th IEEE International Conference on Internet of People. Leicester.

[75] Kieran Woodward, Eiman Kanjo, David Brown, T. M. McGinnity, Becky Inkster, Donald J Macintyre, and Athanasios Tsanas. 2020. Beyond Mobile Apps: A Survey of Technologies for Mental Well-being. IEEE Transactions on Affective Computing (2020).

[76] Kieran Woodward, Eiman Kanjo, Andreas Oikonomou, and Samuel Burton. 2018. Emoecho: A tangible interface to convey and communicate emotions. In UbiComp/ISWC 2018 - 2018 ACM International foint Conference on Pervasive and Ubiquitous Computing and Proceedings of the 2018 ACM International Symposium on Wearable Computers.

[77] Kieran Woodward, Eiman Kanjo, Muhammad Umir, and Corina Sas. 2019. Harnessing Digital Phenotyping to Deliver Real-Time Interventional Bio-Feedback. In WellComp'19: 2nd International Workshop on Computing for Well-Being UBICOMP

[78] Eman M. G. Younis, Eiman Kanjo, and Alan Chamberlain. 2019. Designing and evaluating mobile self-reporting techniques: crowdsourcing for citizen science. Personal and Ubiquitous Computing (mar 2019), 1-10.

\section{ACKNOWLEDGMENTS}

This project has been funded by the Nurture Network (eNurture). eNurture is fundedby UK Research and Innovation (UKRI) and their support is gratefully acknowledged (Grant reference: ES/S004467/1). Any views expressed here are those of the project investigators and do not necessarily represent the views of eNurture or UKRI. We wish to thank all members of the NICER group for their participation in this in the co-design workshops, data collection trials and subsequent focus groups. 